\title{
Individual patterns in aging: monkey's lessons
}

\author{
Vladimir N. Anisimov
}

N.N. Petrov Research Institute of Oncology, Pesochny-2, St. Petersburg 197758, Russia

Commentary on: Goncharova et al. Aging of the hypothalamic-pituitary-adrenal axis in nonhuman primates with depressionlike and aggressive behavior. Aging. 2010; 2: this issue

E-mail: aging@mail.ru

Recent research on rhesus monkeys suggest that the effects of calorie restriction in nonhuman primates recapitulate many of the physiological, hematological, hormonal, immunological, and biochemical effects produced in rodents [1,2]. Measurments of markers of age-related diseases such as diabetes, cardiovascular disease, and possibly cancer suggest that restricted monkeys are healthier than ad libitum counterparts [3]. Thus, studies in monkeys are very important for identification and characterization of candidate biomarkers of aging. At least one biomarker, the rate of decrease in plasma dehydroepiandrosterone (DHEA), suggests that calorie restricted monkeys may be aging slowly [3]. The intrinsic factors, contributing to decreased stress adaptability in old animals, namely disturbances of the hypothalamic-pituitary-adrenal (HPA) axis, are likely to be responsible, at least in part, for health deterioration in the aged [4,5]. At the same time it is well known that the heterogeneity is crucial feature of the aging process. There are individual peculiarities in vulnerability and resistance to stresses and stress-related pathologies among different persons due to the heterogeneity of the ageing process. It is of great importance to elucidate the individual specificity of age-related changes of the HPA axis in the context of aging of the organism as a whole and the stressdependent age pathologies. A paper by Goncharova and colleagues from Research Institute of Medical Primatology at Sochi, Russia [6] renders further support to this important issue and indicate a number of highly relevant points that will have to be taken into consideration for a better understanding of mechanisms involved and possible extrapolations to the situation in humans. Their aim was see in which way the differences in the HPA axis function in young (6-8 years) and old (20-27 years) female rhesus monkeys depends on various behavioral types, under basal conditions, as well as under conditions of acute psychoemotional stress. It has been found that the monkeys with depression-like behavior demonstrate age-related changes in the HPA axis function, accompanied by maximal absolute and relative hypercortisolemia under basal conditions as well as under stress. The authors have also found that young aggressive monkeys, in comparison with young monkeys of other behavior groups, demonstrated the highest plasma levels of DHEA sulfate and the lowest molar ratios between cortisol and DHEA sulfate. No such differences were observed between old aggressive animals and other behavioral groups. The least pronounced age-related changes in the HPA axis have been revealed for the monkeys with average behavior. Goncharova and colleagues have found, for the first time, that monkeys with depressionlike behavior demonstrate age-related changes in HPA axis function that are accompanied by maximal absolute and relative hypercortisolemia under basal conditions, as well as by a significantly greater increase in plasma cortisol levels under acute stress. It worthy to note that taken together these results suggest that age-related dysfunctions of the HPA axis are individual features associated with peculiarities of adaptive behavior of animals. This approach seems very fruitful for future studies. Indeed, nonhuman primates and humans are in physiology of HPA: both species have cortisol as a main glucocorticoid hormone, similar circadian rhythm of HPA activity, age-related decline in DNEA sulfate secretion [7-9]. Furthermore, unlike rodents, nonhuman primates feature the psycho-emotional reactions and adaptive behavior more similar to those in humans. Thus, Goncharova and colleagues studies focused on basic and complex issues which are important for better understanding of individual peculiarities of human aging. 


\section{REFERENCES}

1. Anderson RM et al. Caloric restriction and aging: studies in mice and monkeys. Toxicol Pathol. 2009; 37:47-51.

2. Spindler SR. Caloric restriction: from soup to nuts. Ageing Res Rev. 2010; 9:324-353.

3. Roth GS et al. Biological effects of caloric restriction in primates. Adv Gerontol. 1999; 3:116-120.

4. Lupien SJ et al. Effects of stress throughout the lifespan on the brain, behaviour and cognition. Nature Rev Neurosci. 2009; 10:434-445.

5. Smith RG et al. Molecular endocrinology and physiology of the aging central nervous system. Endocr Rev. 2005; 26:203-250.

6. Goncharova ND et al. Aging of the hypothalamic-pituitaryadrenal axis in nonhuman primates with depression-like and aggressive behavior. Aging. 2010; 2: this issue

7. Goncharova ND and Lapin BA. Effects of aging on hypothalamic-pituitary-adrenal system function in non-human primates. Mech Ageing Dev. 2002; 123:1191-1201.

8. Goncharova ND and Lapin BA. Age-related endocrine dysfunction in nonhuman primates. Ann NY Acad Sci. 2004; 1019:321-325.

9. Kemnitz JW et al. Serum dehydroepiandrosterone sulfate concentrations across the life span of laboratory-housed rhesus monkeys. J Med Primatol. 2000; 29:330-337. 